\title{
Retraction Note to: MicroRNA-134 Promotes the Development of Atherosclerosis Via the ANGPTL4/LPL Pathway in Apolipoprotein E Knockout Mice
}

\author{
Qiong Ye ${ }^{1,2,6}$, Guo-Ping Tian², Hai-Peng Cheng ${ }^{1}$, Xin Zhang ${ }^{1}$, Xiang Ou ${ }^{3}$, Xiao-Hua Yu ${ }^{1}$, Ru-Qi Tan², \\ Feng-Yun Yang ${ }^{2}$, Duo Gong ${ }^{1}$, Chong Huang ${ }^{1}$, Yan-Jun Pan ${ }^{2}$, Jie Zhang ${ }^{4}$, Ling-Yan Chen ${ }^{1}$, Zhen-Wang Zhao ${ }^{1}$, \\ Wei Xie ${ }^{1}$, Liang Li ${ }^{1}$, Min Zhang ${ }^{1}$, Xiao-Dan Xia ${ }^{1}$, Xi-Long Zheng ${ }^{5}$ and Chao-Ke Tang ${ }^{1}$ \\ ${ }^{1}$ Institute of Cardiovascular Research, Key Laboratory for Atherosclerology of Hunan Province, Medical Research Center, Hunan \\ Province Cooperative Innovation Center for Molecular Target New Drug Study, University of South China, Hunan, China \\ ${ }^{2}$ Department of Cardiovascular Medicine, The Second Affiliated Hospital of University of South China, Hunan, China \\ ${ }^{3}$ Department of Endocrinology, The First Hospital of Changsha, Changsha, China \\ ${ }^{4}$ Department of Spinal Surgery, The Second Affiliated Hospital of University of South China, Hunan, China \\ ${ }_{5}^{5}$ Department of Biochemistry and Molecular Biology, Libin Cardiovascular Institute of Alberta, Cumming School of Medicine, \\ University of Calgary, Health Sciences Center, 3330 Hospital Dr NW, Calgary, Alberta, Canada \\ ${ }^{6}$ Department of Ultrasound, Huadu District People’s Hospital of Guangzhou, Guangdong, China
}

Address for correspondence: Chao-Ke Tang, Institute of Cardiovascular Research, University of South China, Hengyang, Hunan 421001, China

\section{Retraction Note to:}

J Atheroscler Thromb, 2018; 25: 244-253

https://doi.org/10.5551/jat.40212

The Editorial Team of the Journal of Atherosclerosis and Thrombosis has recently confirmed that some data in the paper written by Qiong Ye et al., published in the Journal (J Atheroscler Thromb 2018; 25: 244-253), including the images of the mouse aorta and the graphs (Fig. 1), are incorrect. Therefore, we have had to take the decision to retract the paper from the Journal of Atherosclerosis and Thrombosis with the agreement from the authors.

Yujiro Asada, Editor-in-Chief, Journal of Atherosclerosis and Thrombosis

Published online: October 1, 2021

Copyright@2021 Japan Atherosclerosis Society

This article is distributed under the terms of the latest version of CC BY-NC-SA defined by the Creative Commons Attribution License. 